\title{
Evolución y tratamiento de los trastornos de la conducta alimentaria en adolescentes: serie de casos
} Treatment of eating disorders in adolescents: case series

\author{
Dra. María B. Saad ${ }^{a}$, Dr. Alfredo Eymann ${ }^{a}$, Dra. Natalia Granados ${ }^{b}$, Dra. Gisela Rotblat ${ }^{c}$, \\ Mg. Soledad Dawson', Mg. Alicia Cibeirac y Dra. Marcela C. Paz ${ }^{a}$
}

\begin{abstract}
RESUMEN
Los trastornos de la conducta alimentaria son enfermedades de elevada prevalencia en la adolescencia y tienen repercusión en la salud integral. El objetivo fue describir su evolución y tratamiento en un grupo de adolescentes. Se estudiaron adolescentes menores de 18 años con al menos 6 meses de seguimiento y tratados por un equipo interdisciplinario.

Se incluyeron 41 adolescentes: 23 presentaron anorexia nerviosa; 9 , trastornos alimentarios no especificados; 7 , bulimia nerviosa y 2, trastorno por atracones. El $35 \%$ de pacientes con anorexia nerviosa requirió internación por complicaciones de la desnutrición. El $69 \%$ de las pacientes con anorexia nerviosa, el $57 \%$ de quienes tenían bulimia nerviosa y el $78 \%$ de quienes tenían un trastorno alimentario no especificado presentaron remisión total o parcial, y no hubo pacientes fallecidos. La mayoría presentó una evolución favorable con el tratamiento. Palabras clave: trastornos alimentarios, adolescencia, grupo de salud interdisciplinario, internación.
\end{abstract}

\begin{abstract}
Eating disorders arehighly prevalent diseases in adolescenceand have an impact on overall health. The objective was to describe the evolution and treatment of eating disorders in adolescents. Adolescents under 18 years of age with at least 6 months of follow-up and treated by an interdisciplinary team were studied. Forty one adolescents were included, 23 presented anorexia nervosa, 9 unspecified eating disorders, 7 bulimia nervosa and 2 binge eating disorders. Thirty five percent of patients with anorexia nervosa required hospitalization for complications of malnutrition. Sixty nine percent of the patients with anorexia nervosa, $57 \%$ with bulimia nervosa, and $78 \%$ with unspecified eating disorders had total or partial remission and there were no deceased patients. The majority presented a favorable evolution with the treatment.
\end{abstract}

Key words: feeding disorders, adolescence, patient care team, hospitalization.

a. Sección de Adolescencia, Servicio de Clínica Pediátrica, Departamento de Pediatría.

b. Sección de Nutrición Pediátrica, Servicio de Clínica Pediátrica, Departamento de Pediatría.

c. Servicio de Salud Mental Pediátrica, Departamento de Pediatría.

Hospital Italiano de Buenos Aires, Ciudad Autónoma de Buenos Aires, Argentina.

Correspondencia:

Dra. Marcela C. Paz: marcela.paz@hospitalitaliano.org.ar

Financiamiento: Ninguno.

Conflicto de intereses: Ninguno que declarar.

Recibido: 25-11-2020

Aceptado: 26-2-2021 http:/ / dx.doi.org/10.5546/ aap.2021.e364

Cómo citar: Saad MB, Eymann A, Granados N, Rotblat G, et al Evolución y tratamiento de los trastornos de la conducta alimentaria en adolescentes: serie de casos. Arch Argent Pediatr 2021;119(4):e364-e369.

\section{INTRODUCCIÓN}

Los trastornos de la conducta alimentaria (TCA) son enfermedades complejas que afectan principalmente a mujeres adolescentes y jóvenes. Se caracterizan por excesiva preocupación por el peso, la figura y la alimentación, y van acompañados de prácticas inadecuadas para el control de los mismos. Son un conjunto de entidades integrado por la anorexia nerviosa (AN), la bulimia nerviosa (BN), los trastornos alimentarios no especificados (TANE) y el trastorno por atracón (BED, por su sigla en inglés de binge eating disorder). ${ }^{1}$

Los TCA producen gran impacto en la salud integral, evidenciado por un marcado deterioro físico y compromiso psiquiátrico que pueden conducir a la muerte. El diagnóstico temprano y el tratamiento oportuno son determinantes para mejorar el pronóstico. ${ }^{2,3}$

Los TCA tienen una distribución y prevalencias variables en diferentes países. En una revisión sistemática de TCA en América Latina, que incluyó 2 estudios argentinos, la tasa de prevalencia fue el 3,5\% BED, el 1,1 \% BN y el $0,1 \%$ AN. $^{4}$

Se ha establecido que en la etiología y patogenia participan factores biológicos, psicológicos, familiares y socioculturales; todos estos factores deben ser considerados para el tratamiento. Asimismo, se propone que los profesionales actuantes tengan experiencia en la atención de adolescentes, en TCA y en trabajo interdisciplinario. ${ }^{5}$

Nos planteamos como objetivo describir la evolución y tratamiento de TCA en adolescentes debido a que no hemos encontrado publicados, en nuestro medio, estudios que indaguen estos aspectos. 


\section{POBLACIÓN Y MÉTODOS}

Se realizó un estudio descriptivo de corte transversal. La población fueron adolescentes con diagnóstico de TCA menores de 18 años con al menos 6 meses de seguimiento y tratamiento por un equipo interdisciplinario. La muestra se obtuvo luego de la revisión de las historias clínicas electrónicas realizada por una médica especialista en adolescencia.

Se utilizó la clasificación del Manual diagnóstico y estadístico de los trastornos mentales, cuarta edición (DSM IV, por su sigla en inglés) para definir los siguientes conceptos:

Anorexia nerviosa: es la negativa a mantener un peso mínimo para la edad y la talla, intenso temor a aumentar de peso, distorsión de la imagen corporal y amenorrea.

Bulimia nerviosa: episodios recurrentes de compulsiones alimentarias (atracones) a compañados de comportamientos compensatorios inadecuados y recurrentes, con una frecuencia mínima de 2 veces por semana durante 3 meses, y que no cumple los criterios de la AN.

Trastorno por atracón: episodios recurrentes de atracones sin comportamientos compensatorios con una frecuencia mínima de 2 veces por semana durante 6 meses.

Trastornos alimentarios no específicos: síntomas de algún trastorno alimentario sin cumplir los criterios específicos de ninguna categoría. ${ }^{1}$

Se definió como equipo interdisciplinario (EI) a un grupo de profesionales integrado por médicas pediatras especialistas en adolescencia, médicas pediatras nutricionistas infantiles, médicas psiquiatras infantojuveniles, psicólogas de familia y psicólogas individuales (Anexo).

Se analizaron las siguientes variables: sexo, edad de inicio de síntomas e inicio del tratamiento, tratamiento previo, número de internaciones, días de internación, atracones, vómitos, ayuno, ejercicio, laxantes, conductas auto-y heteroagresivas (golpes, cortes autoprovocados, violencia verbal y física hacia otros), ideación suicida, uso de anticonceptivos orales (ACO), fluctuaciones a otro diagnóstico de TCA y evolución clínica. En el subgrupo de pacientes con AN se evaluó el índice de masa corporal (IMC) y puntaje $\mathrm{Z}$ al inicio, a los 6 meses y al año de tratamiento.

Se definieron las siguientes condiciones para evaluar la evolución clínica a los 6 y a los 12 meses de tratamiento: para AN, recuperación del IMC, remisión del miedo a engordar y desaparición de la distorsión de la imagen corporal (la amenorrea no fue considerada ya que varias pacientes recibieron $\mathrm{ACO})$. Para $\mathrm{BN}$, remisión de los atracones, de las conductas compensatorias y estabilidad emocional. Para TANE, recuperación del IMC (si hubiera estado alterado), remisión de las conductas alimentarias inadecuadas y estabilidad emocional; para BED, remisión de los atracones y estabilidad emocional.

Se definieron las siguientes categorías de evolución clínica: remisión total cuando se cumplieron las 3 condiciones para $A N$, $\mathrm{BN}$ y TANE, y las 2 condiciones para BED; remisión parcial al cumplimiento de 1 o 2 de las condiciones, y sin cambios cuando continuaron las mismas condiciones.

Se definieron las siguientes categorías para conductas de seguimiento: continuación del tratamiento, alta (remisión total durante al menos 12 meses), derivación a otro equipo y abandono de tratamiento.

El estudio fue aprobado por el Comité de Ética y Protocolos de Investigación de nuestra institución (N. . 5215).

\section{RESULTADOS}

Se incluyeron 41 adolescentes (100\% de las historias clínicas revisadas). La AN fue el TCA más frecuente (23 pacientes), fue el grupo de adolescentes que requirió internación y tuvo el único paciente varón (Tabla 1).

Los vómitos y ejercicios fueron las conductas de control del peso más utilizadas y los atracones y las conductas autoagresivas fueron las conductas impulsivas más frecuentes (Tabla 1).

La Tabla 2 muestra que la mediana del IMC inicial de los adolescentes con AN que requirieron internación fue 15,1 y de los que recibieron tratamiento ambulatorio, 17,4. Ambos grupos presentaron una mejoría a los 6 y a los 12 meses de tratamiento.

La Tabla 3 describe las características de los ciclos menstruales en pacientes con AN y TANE. La mediana y rango intercuartílico (RIC) de amenorrea fue 6 (5 -14) meses y la recuperación de los ciclos menstruales ocurrió con un puntaje $\mathrm{Z}$ de IMC -1.

La Tabla 4 describe la evolución clínica y la conducta de seguimiento de los pacientes. La mayoría tuvo una mejoría total o parcial, y continuó su tratamiento. No hubo pacientes fallecidos.

Durante la duración del estudio, 4 pacientes 
TABla 1. Características demográficas, tratamientos, conductas de control de peso e impulsivas de los pacientes con trastornos de la conducta alimentaria

\begin{tabular}{|c|c|c|c|c|c|}
\hline & & \multicolumn{4}{|c|}{ Diagnóstico } \\
\hline & & AN & TANE & BN & BED \\
\hline & & 23 & 9 & 7 & 2 \\
\hline Mujeres & $\mathrm{n}(\%)$ & $22(95,6)$ & $9(100)$ & $7(100)$ & $2(100)$ \\
\hline Hombres & $\mathrm{n}(\%)$ & $1(4,3)$ & $0(0)$ & $0(0)$ & $0(0)$ \\
\hline Edad inicio síntomas (años) & mediana (RIC) & $13(12-15)$ & $14,5(14-15,5)$ & $15(14-16)$ & $13(12-14)$ \\
\hline Tratamiento previo & $\mathrm{n}(\%)$ & $10(43,4)$ & $4(44,4)$ & $1(14,2)$ & $1(50)$ \\
\hline Requirió internación & $\mathrm{n}(\%)$ & $8(34,7)$ & $0(0)$ & $0(0)$ & $0(0)$ \\
\hline Internación (días) & mediana (RIC) & $49,5(40,5-61,5)$ & $0(0)$ & $0(0)$ & $0(0)$ \\
\hline Vómitos & $\mathrm{n}(\%)$ & $4(17,3)$ & $1(11,1)$ & $7(100)$ & $0(0)$ \\
\hline Ejercicio & $\mathrm{n}(\%)$ & $8(34,7)$ & $2(22,2)$ & $2(29)$ & $2(100)$ \\
\hline Ayuno & $\mathrm{n}(\%)$ & $3(13)$ & $0(0)$ & $0(0)$ & $0(0)$ \\
\hline Laxantes & $\mathrm{n}(\%)$ & $0(0)$ & $1(11,1)$ & $2(29)$ & $1(50)$ \\
\hline Atracones & $\mathrm{n}(\%)$ & $4(17,3)$ & $2(22,2)$ & $7(100)$ & $2(100)$ \\
\hline Conductas autoagresivas & $\mathrm{n}(\%)$ & $5(21,7)$ & $3(33,3)$ & $1(14,2)$ & $1(50)$ \\
\hline Conductas heteroagresivas & $\mathrm{n}(\%)$ & $1(4,3)$ & $1(11,1)$ & $0(0)$ & $0(0)$ \\
\hline Ideación suicida & $\mathrm{n}(\%)$ & $3(13)$ & $1(11,1)$ & $0(0)$ & $1(50)$ \\
\hline
\end{tabular}

AN: anorexia nerviosa, TANE: trastorno alimentario no especificado, BN: bulimia nerviosa, BED: trastorno por atracón, RIC: rango intercuartílico.

TABLA 2. Evolución de peso en pacientes con anorexia nerviosa en seguimiento ambulatorio o requerimiento de internación

\begin{tabular}{|c|c|c|c|c|c|}
\hline & & \multicolumn{2}{|c|}{ Seguimiento ambulatorio } & \multicolumn{2}{|c|}{ Requerimiento de internación } \\
\hline & & IMC & Puntaje Z & IMC & Puntaje Z \\
\hline Peso inicial & $\begin{array}{l}\text { mediana } \\
\text { (RIC) }\end{array}$ & $\begin{array}{c}17,4 \\
(15,4-18,2)\end{array}$ & $\begin{array}{c}-1 \\
(-2,1-0,5)\end{array}$ & $\begin{array}{c}15,1 \\
(14,8-17)\end{array}$ & $\begin{array}{c}-1,9 \\
(-3,3--1)\end{array}$ \\
\hline Peso a los 6 meses & $\begin{array}{l}\text { mediana } \\
(\text { RIC) }\end{array}$ & $\begin{array}{c}18,7 \\
(18,4-19,2)\end{array}$ & $\begin{array}{c}-0,1 \\
(-0,7-0,2)\end{array}$ & $\begin{array}{c}16,8 \\
(16,3-17,5)\end{array}$ & $\begin{array}{c}-1,2 \\
(-1,6--0,7)\end{array}$ \\
\hline Peso a los 12 meses & $\begin{array}{l}\text { mediana } \\
(\text { RIC) }\end{array}$ & $\begin{array}{c}18,7 \\
(18,6-23)\end{array}$ & $\begin{array}{c}-0,7 \\
(-0,8-0,9)\end{array}$ & $\begin{array}{c}17,3 \\
(15,8-17,5)\end{array}$ & $\begin{array}{c}-1,1 \\
(-2,4--1,1)\end{array}$ \\
\hline
\end{tabular}

IMC, índice de masa corporal, RIC: rango intercuartílico.

TABLA 3. Ciclos menstruales en pacientes con anorexia nerviosa y trastornos alimentarios no especificados

\begin{tabular}{|c|c|c|c|}
\hline & & \multicolumn{2}{|c|}{ Diagnóstico } \\
\hline & & $\begin{array}{c}\text { AN } \\
22\end{array}$ & $\begin{array}{c}\text { TANE } \\
9\end{array}$ \\
\hline \multicolumn{2}{|l|}{ Menarca } & $18(81,8)$ & $9(100)$ \\
\hline Amenorrea & $\begin{array}{l}\text { Primaria } \\
\text { Secundaria }\end{array}$ & $\begin{array}{c}3(13,6) \\
19(86,3)\end{array}$ & $\begin{array}{c}0(0) \\
1(11,1)\end{array}$ \\
\hline Indicación de ACO & $\begin{array}{l}\text { RS } \\
\text { Estímulo }\end{array}$ & $\begin{array}{l}5(22,7) \\
3(13,6)\end{array}$ & $\begin{array}{c}1(11,1) \\
0(0)\end{array}$ \\
\hline \multicolumn{2}{|c|}{ Inicio de relaciones sexuales $\mathrm{n}(\%)$} & $5(22,7)$ & $1(11,1)$ \\
\hline \multicolumn{2}{|c|}{ Recuperación menstruación espontánea n $(\%)$} & $11(57,8)$ & $0(0)$ \\
\hline \multicolumn{2}{|c|}{ Puntaje Z al recuperar ciclos menstruales (mediana, RIC) } & $(-1,6--1,1)$ & $0(0)$ \\
\hline \multicolumn{2}{|c|}{ Porcentaje de peso ideal al recuperar ciclos menstruales $\mathrm{n}(\%)$} & $11(93,7)$ & $0(0)$ \\
\hline
\end{tabular}

AN: anorexia nerviosa, TANE: trastorno alimentario no especificado, RS: relaciones sexuales, ACO: anticonceptivos orales, RIC: rango intercuartílico. 
con AN fluctuaron a otros diagnósticos: 1 a TANE, 1 a BN y 2 a BED. Un solo paciente con BN fluctuó a BED y no se observaron fluctuaciones desde otros diagnósticos a AN.

\section{DISCUSIÓN}

Nuestro estudio mostró que la mayoría de los pacientes presentó AN y tuvieron una buena evolución con el tratamiento de un EI. El $56 \%$ de nuestra muestra presentó AN; es conocido que la prevalencia en la población general es menor, pero estos pacientes suelen presentar un importante compromiso clínico, nutricional y psicopatológico y requieren ser derivados a instituciones con experiencia en estas patologías. ${ }^{6,7}$

La fluctuación de $\mathrm{AN}$ a otros diagnósticos fue menor a lo descrito por Tozzi, que encontró que el $36 \%$ de AN fluctuó a BN dentro de los 5 años de enfermedad. ${ }^{8}$

Las conductas auto- y heteroagresivas se presentaron en mayor medida que en la población sin TCA. Diferentes autores han descrito que estas conductas están relacionadas con signos de impulsividad e intentos inadecuados de regulación emocional; asimismo, las emociones más prevalentes son insatisfacción con la imagen corporal, culpa, baja autoestima, frustración y tristeza. ${ }^{9,10}$

Nuestro estudio mostró que los adolescentes con AN presentaron mayor ideación suicida a diferencia del estudio de Swanson, que la asocia a BN y BED. ${ }^{5}$

Durante el seguimiento, ningún paciente presentó intentos de suicidio ni falleció. Otros estudios han descrito una mortalidad del $5 \%$ por suicidio y alteraciones del medio interno, y que el riesgo de muerte se incrementó a medida que aumentó el tiempo de seguimiento., ${ }^{9,11}$

La amenorrea en adolescentes con AN es consecuencia de alteraciones en el eje hipotálamohipófiso-gonadal. Las pacientes con AN que no tomaban ACO recuperaron espontáneamente los ciclos menstruales cuando el peso alcanzó una mediana de peso ideal del $93,7 \%$ y puntaje $Z$ de IMC -1. Estos valores son ligeramente superiores a otros estudios que refieren que la recuperación de los ciclos menstruales en alrededor del $85 \%$ de las pacientes se logró con un peso cercano al $90 \%$ del peso ideal. En cuanto al tiempo, la recuperación de los ciclos menstruales puede demorar entre 6 y 18 meses luego de recuperar el peso adecuado. ${ }^{12,13}$

Los adolescentes de nuestro estudio presentaron buena evolución clínica y similares resultados a un metaanálisis, donde el $47 \%$ de los pacientes con AN lograron recuperación completa; el $33 \%$, parcial, y el $20 \%$ evolucionó a cronicidad. Los referidos a BN mostraron que el $48 \%$ se recuperó en forma completa; el $26 \%$, parcial, y el $26 \%$ evolucionó a cronicidad. ${ }^{14}$ Otros estudios con seguimientos prolongados mostraron resultados similares. ${ }^{11,15}$

Este estudio presenta las siguientes limitaciones: se desarrolló en un solo centro asistencial, los datos fueron relevados de una historia clínica electrónica, los criterios de diagnóstico se basaron en el DSM IV y el tiempo de seguimiento fue de algunos meses. Sin embargo, consideramos que muestra resultados valiosos con una estrategia de tratamiento interdisciplinaria de una enfermedad compleja en la adolescencia.

\section{CONCLUSIONES}

La mayoría de los pacientes presentó AN y un tercio de estos requirió internación. La mayoría de los pacientes presentó una evolución favorable con el tratamiento.

TABLA 4. Evolución clínica y conducta de seguimiento en los pacientes con trastornos de la conducta alimentaria

\begin{tabular}{|c|c|c|c|c|c|}
\hline & & \multicolumn{4}{|c|}{ Diagnóstico } \\
\hline & & $\begin{array}{c}\text { AN } \\
23\end{array}$ & $\begin{array}{c}\text { TANE } \\
9\end{array}$ & $\begin{array}{c}\mathrm{BN} \\
7\end{array}$ & $\begin{array}{c}\text { BED } \\
2\end{array}$ \\
\hline Remisión total & $\mathrm{n}(\%)$ & $6(26)$ & $2(22,2)$ & $2(28,7)$ & $0(0)$ \\
\hline Remisión parcial & $\mathrm{n}(\%)$ & $10(43,4)$ & $5(55,5)$ & $2(28,5)$ & $0(0)$ \\
\hline Sin cambios & $\mathrm{n}(\%)$ & $7(30,4)$ & $2(22,2)$ & $3(42,8)$ & $2(100)$ \\
\hline Alta & $\mathrm{n}(\%)$ & $2(8,6)$ & $2(22,2)$ & $1(14,2)$ & $0(0)$ \\
\hline Continuó tratamiento & $\mathrm{n}(\%)$ & $20(86,9)$ & $6(66,6)$ & $4(57,1)$ & $1(50)$ \\
\hline Derivada & $\mathrm{n}(\%)$ & $1(4,3)$ & $0(0)$ & $0(0)$ & $0(0)$ \\
\hline Abandono & $\mathrm{n}(\%)$ & $0(0)$ & $1(11,1)$ & $2(28,5)$ & $1(50)$ \\
\hline
\end{tabular}

AN: anorexia nerviosa, TANE: trastorno alimentario no especificado, BN: bulimia nerviosa, BED: trastorno por atracón. 


\section{REFERENCIAS}

1. Comité del DSM-IV. Manual diagnóstico y estadístico de los trastornos mentales. Barcelona: Masson; 1995.

2. España. Ministerio de Salud y Consumo. Guía de práctica clínica sobre trastornos de la conducta alimentaria. Madrid: Agéncia D' Avaluació de Tecnología: Recerca Médiques de Cataluña; 2009.

3. Campbell K, Peebles R. Eating disorders in children and adolescents: state of the art review. Pediatrics. 2014; 134(3):582-92.

4. Kolar DR, Mejía Rodríguez DL, Chams MM, Hoek HW. Epidemiology of eating disorders in Latin America: a systematic review and meta-analysis. Curr Opin Psychiatry. 2016; 29(6):363-71.

5. Stewart TM, Williamson DA. Multidisciplinary treatment of eating disorders. Part 2: primary goals and content of treatment. Behav Modif. 2004; 28(6):831-53.

6. Hornberger LL, Margo A. Lane. Identification and Management of Eating Disorders in Children and Adolescents. Pediatrics. 2021; 147(1):e2020040279.

7. SwansonSA, CrowSJ, LeGrangeD, SwendsenJ, Merikangas KR. Prevalence and correlates of eating disorders in adolescents: results from the national comorbidity survey replication adolescent supplement. Arch Gen Psychiatry. $2011 ; 68(7): 714-23$.
8. Tozzi F, Thornton LM, Klump KL, Fichter MM, et al. Symptom fluctuation in eating disorders: correlates of diagnostic crossover. Am J Psychiatry. 2005; 162(4):732-40.

9. Martínez Baquero LC, Vianchá Pinzón MA, Pérez Prada $\mathrm{MP}$, Avendaño Prieto BL. Asociación entre conducta suicida y síntomas de anorexia y bulimia nerviosa en escolares de Boyacá, Colombia. Acta Colomb Psicol. 2017; 20(2):178-88.

10. Varela-Besteiro O, Serrano-Troncoso E, Rodríguez-Vicente V, Curet-Santisteban M, et al. Ideación suicida y conductas autolesivas en adolescentes con Trastornos de la Conducta Alimentaria. Actas Esp Psiquiatr. 2017; 45(4):157-66.

11. Steinhausen HC. Outcome of eating disorders. Child Adolesc Psychiatr Clin N Am. 2009; 18(1):225-42.

12. Jacoangeli F, Masala S, Staar Mezzasalma F, Fiori R, et al. Amenorrhea after weight recover in anorexia nervosa: role of body composition and endocrine abnormalities. Eat Weight Disord. 2006; 11(1):e20-6.

13. Schorr M, Miller KK. The endocrine manifestations of anorexia nervosa: mechanisms and management. Nat Rev Endocrinol. 2017; 13(3):174-86.

14. Jagielska G, Kacperska I. Outcome, comorbidity and prognosis in anorexia nervosa. Psychiatr Pol.2017;51(2):20518.

15. Rosen DS, American Academy of Pediatrics Committee on Adolescence. Pediatrics. 2010; 126(6):1240-53. 
ANEXO

\begin{tabular}{|c|c|c|c|}
\hline Integrantes & Roles & Ámbitos & Procesos asistenciales \\
\hline $\begin{array}{l}\text { Clinica } \\
\text { Pediátrica } \\
\text { Adolescencia }\end{array}$ & $\begin{array}{l}\text { Evaluar la salud integral del } \\
\text { adolescente } \\
\text { Definir el ámbito del tratamiento } \\
\text { Contribuir al diagnóstico de TCA } \\
\text { Detectar complicaciones y riesgo } \\
\text { Prescribir estudios } \\
\text { Considerar la necesidad de limitar la } \\
\text { actividad } \\
\text { Evaluar el desarrollo del tratamiento }\end{array}$ & \multirow{5}{*}{$\begin{array}{l}\text { Ambulatorio: } \\
\text { consultorios } \\
\text { externos } \\
\\
\text { Internación: } \\
\text { sala general y } \\
\text { unidad de } \\
\text { cuidados } \\
\text { intensivos }\end{array}$} & \multirow{2}{*}{$\begin{array}{l}\text { Trabajo interdisciplinario en reuniones } \\
\text { semanales: } \\
\text { Atención integral centrada en el paciente } \\
\text { Aporte de cada disciplina } \\
\text { Intercambio de información } \\
\text { Integración de los aportes } \\
\text { Articulación para lograr consensos } \\
\text { Elaboración del diagnóstico } \\
\text { Definición de metas y estrategias } \\
\text { Información unificada para compartir con el } \\
\text { paciente y la familia } \\
\text { Asistencia: simultánea y secuencial en los } \\
\text { diferentes ámbitos en el manejo inicial y } \\
\text { seguimiento, y con una frecuencia diaria en } \\
\text { pacientes graves }\end{array}$} \\
\hline $\begin{array}{l}\text { Nutrición } \\
\text { Pediátrica }\end{array}$ & $\begin{array}{l}\text { Evaluar el estado nutricional y la } \\
\text { conducta alimentaria } \\
\text { Contribuir al diagnóstico del TCA } \\
\text { Diseñar e implementar el plan } \\
\text { nutricional } \\
\text { Abordar los comportamientos } \\
\text { relacionados con la comida y el comer. } \\
\text { Favorecer la modificación de la rigidez } \\
\text { cognitiva } \\
\text { Evaluar el desarrollo del tratamiento }\end{array}$ & & \\
\hline $\begin{array}{l}\text { Psiquiatría } \\
\text { Infantil }\end{array}$ & $\begin{array}{l}\text { Evaluar conductas de riesgo de auto- y } \\
\text { heteroagresiones } \\
\text { Formular el diagnóstico } \\
\text { psicopatológico y contribuir al } \\
\text { diagnóstico del TCA } \\
\text { Evaluar la psicopatología subyacente } \\
\text { Evaluar la necesidad de medicación } \\
\text { psicofarmacológica } \\
\text { Evaluar el desarrollo del tratamiento }\end{array}$ & & $\begin{array}{l}\text { Vias de comunicación: personal, grupal, } \\
\text { correo electrónico, teléfono celular y grupo } \\
\text { de WhatsApp }\end{array}$ \\
\hline $\begin{array}{l}\text { Psicología } \\
\text { Individual }\end{array}$ & $\begin{array}{l}\text { Buscar la alianza terapéutica con el } \\
\text { paciente y su familia } \\
\text { Formular el diagnóstico individual y } \\
\text { contribuir al diagnóstico del TCA } \\
\text { Psicoterapia individual } \\
\text { Evaluar el desarrollo del tratamiento }\end{array}$ & & $\begin{array}{l}\text { Fluida comunicación } \\
\text { Respeto entre las profesionales } \\
\text { Excelente clima de trabajo } \\
\text { Accesibilidad de otras especialidades } \\
\text { Apoyo institucional }\end{array}$ \\
\hline $\begin{array}{l}\text { Psicologia } \\
\text { Familiar }\end{array}$ & $\begin{array}{l}\text { Buscar la alianza terapéutica con el } \\
\text { paciente y su familia } \\
\text { Formular un diagnóstico familiar y } \\
\text { contribuir al diagnóstico del TCA } \\
\text { Psicoterapia familiar } \\
\text { Evaluar el desarrollo del tratamiento }\end{array}$ & & $\begin{array}{l}\text { Debilidades: } \\
\text { No disponibilidad de sala especifica } \\
\text { Limitaciones de financiamiento }\end{array}$ \\
\hline
\end{tabular}

TCA: trastorno de conducta alimentaria. 